\title{
Phase transitions in bismuth-modified silver niobate ceramics for high power energy storage
}

Ye Tian, ${ }^{1,2}$ Li Jin, ${ }^{1}$ Hangfeng Zhang, ${ }^{3}$ Zhuo Xu,${ }^{1}$ Xiaoyong Wei,${ }^{1 *}$ Giuseppe Viola, ${ }^{2,4}$

Isaac Abrahams, ${ }^{3}$ Haixue Yan $^{2 *}$

${ }^{1}$ Electronic Materials Research Laboratory, Key Laboratory of the Ministry of Education \& International Center for Dielectric Research, Xi'an Jiaotong University, Xian 710049, China

${ }^{2}$ School of Engineering and Materials Science, Queen Mary University of London, Mile End Road, London E1 4NS, United Kingdom

${ }^{3}$ School of Biological and Chemical Sciences, Queen Mary University of London, Mile End Road, London E1 4NS, United Kingdom

${ }^{4}$ Department of Applied Science and Technology, Institute of Materials Physics and Engineering, Corso Duca degli Abruzzi 24, 10129 Torino, Italy

Corresponding Authors*

E-mail: wdy@mail.xjtu.edu.cn and h.x.yan@mail.qmul.ac.uk 


\section{Abstract}

Ceramics of composition $\mathrm{Ag}_{1-3 x} \mathrm{Bi}_{x} \mathrm{NbO}_{3}(0.005 \leq x \leq 0.040)$ were prepared by solid state methods and their structure and electrical behavior were characterized with a view to their potential use as high power energy storage materials. All compositions exhibited an average orthorhombic non-polar structure. The low temperature phase transitions $\mathrm{M}_{1} \leftrightarrow \mathrm{M}_{2}$ and $\mathrm{M}_{2} \leftrightarrow \mathrm{M}_{3}$ and the freezing temperature $T_{\mathrm{f}}$ found in $\mathrm{AgNbO}_{3}$ are increasingly shifted to lower temperatures with increasing $x$-value. Similarly to $\mathrm{AgNbO}_{3}$, the structure of the $\mathrm{M}_{2}$ phase above $T_{\mathrm{f}}$ is antiferroelectric. In the $\mathrm{M}_{2}$ phase, below $T_{f}$ it is proposed that the structure possesses local polar regions, which can expand during electrical loading within an average non-polar antiferroelectric matrix. The polar domains found in the $\mathrm{M}_{1}$ phase of $\mathrm{AgNbO}_{3}$ diminish with increasing bismuth content, as confirmed by the suppression of domain switching peaks in the current-polarization-electric field hysteresis loops. The antiferroelectric to ferroelectric electric field-induced transformation is progressively hindered, as the structure evolves towards long-range antiferroelectric order with increasing bismuth content. Moreover, up to a certain substitution level, bismuth addition is seen to enhance energy storage properties compared to unsubstituted $\mathrm{AgNbO}_{3}$, with a high energy storage density of $2.6 \mathrm{~J} \mathrm{~cm}^{-3}$ and high energy efficiency of 0.86 achieved. These values make these materials amongst the best performing energy storage lead-free ceramics currently known.

Key words: dielectric, energy storage, lead-free, ferroelectrics, antiferroelectrics, $\mathrm{AgNbO}_{3}$ 


\section{Introduction}

Growing demand for high-power density energy storage devices for use in portable electronics, electrical vehicles etc. has led to research into new dielectric energy storage materials that exhibit high efficiency. The high breakdown strength and energy density of dielectric polymers have allowed for their use in capacitors for high-power energy storage. However, polymer capacitors are limited by their application temperature range. Compared to polymers, dielectric ceramics have superior thermal properties, allowing for a wider operational temperature range. For energy storage applications, ferroelectric ceramics with high saturation polarization, low remnant polarization and high dielectric breakdown strength $\left(E_{\mathrm{b}}\right)$ are required. ${ }^{1-3}$ The most utilized ferroelectrics are based on lead containing oxides, ${ }^{4,5}$ but due to concerns over the toxicity of lead, there is a drive to develop $\mathrm{Pb}$-free ferroelectric (FE) ceramics for many applications, including dielectric energy storage $\mathrm{e}^{6-10}$.

Silver niobate $\mathrm{AgNbO}_{3}$ is a candidate material for potential application in energy storage devices. However, despite its high energy density of $2.1 \mathrm{~J} \mathrm{~cm}^{-3}$, it exhibits low energy efficiency, with a relatively large hysteresis observed in ferroelectric hysteresis loops at high field. ${ }^{11,12}$ Structural investigations of $\mathrm{AgNbO}_{3}$ have revealed that at room temperature both $\mathrm{Ag}^{+}$and $\mathrm{Nb}^{5+}$ cations experience antiparallel off-center displacements within their respective polyhedra. ${ }^{13-15}$ However, the existence of polar regions at room temperature was ascertained by detailed diffraction studies has been reported more recently. ${ }^{12,16,17}$ The coexistence of antiferroelectric (AFE) and FE states in $\mathrm{AgNbO}_{3}$ yields a complicated sequence of electric field-induced events, as observed in the current-polarization-electric field hysteresis loops previously reported. $^{12}$

It was found that bismuth niobate, $\mathrm{BiNb}_{3} \mathrm{O}_{9}$, crystalizes as an A-site deficient metastable perovskite super-cell structure (i.e., $\mathrm{Bi}_{1 / 3} \mathrm{NbO}_{3}$ ). ${ }^{18}$ Additionally, the ionic radius $\mathrm{Bi}^{3+}$ is smaller than that of $\mathrm{Ag}^{+}(r=1.17$ and $1.28 \AA$, respectively for the ions in eight coordinate geometry), ${ }^{19}$ which is beneficial for reducing the perovskite tolerance factor and the promotion of antiferroelectricity. ${ }^{20}$ Thus, bismuth might be 
expected to show some solubility in the silver niobate structure and this could be used to improve the energy storage characteristics of $\mathrm{AgNbO}_{3}$. In the present work we report the structure and dielectric properties of ceramics in the $\mathrm{AgNbO}_{3}-\mathrm{Bi}_{1 / 3} \mathrm{NbO}_{3}$ (i.e., $\mathrm{Ag}_{1-3 x} \mathrm{Bi}_{x} \mathrm{NbO}_{3}$ ) pseudo-binary solid solution. Bismuth substitution in this system is shown to enhance energy density and efficiency.

\section{Experimental}

Polycrystalline ceramic samples of $\mathrm{Ag}_{1-3 x} \mathrm{Bi}_{x} \mathrm{NbO}_{3}(\mathrm{ABN} x, 0.005 \leq x \leq 0.040)$ were synthesized by a conventional solid-state process. All starting materials were purchased from Sinopharm Chemical Reagent Co. Ltd., China. Stoichiometric amounts of $\mathrm{Ag}_{2} \mathrm{O}(99.7 \%), \mathrm{Nb}_{2} \mathrm{O}_{5}(99.99 \%)$ and $\mathrm{Bi}_{2} \mathrm{O}_{3}(99.9 \%)$ were ground in ethanol for 12 hours using a planetary ball mill (QM-3SP4, Nanjing University Instrument, China). After drying, the mixtures were placed into alumina porcelain boats and heated to $800{ }^{\circ} \mathrm{C}$, at a heating rate of $5{ }^{\circ} \mathrm{C} \min ^{-1}$, in a tube furnace (GLS-1600X, Hefei Kejing, China) and calcined at this temperature for $4 \mathrm{~h}$, in flowing oxygen at a rate of $500 \mathrm{~cm}^{3} \mathrm{~min}^{-1}$. The calcined powders were re-ground in the ball mill for $4 \mathrm{~h}$ in ethanol. After drying, the powders were mixed with $5 \mathrm{wt} \%$ polyvinyl alcohol (PVA) solution and pressed into pellets with a diameter of $15 \mathrm{~mm}$ and thickness of 1 2 $\mathrm{mm}$ under $400 \mathrm{MPa}$ uniaxial pressure. The pellets were heated at a rate of $5{ }^{\circ} \mathrm{C} \min ^{-1}$ to $600{ }^{\circ} \mathrm{C}$ and held at this temperature for $2 \mathrm{~h}$ to burn off the PVA. The samples were subsequently sintered at temperatures between 1060 and $1090{ }^{\circ} \mathrm{C}$ in oxygen at a flow rate of $500 \mathrm{~cm}^{3} \mathrm{~min}^{-1}$ for $6 \mathrm{~h}$, followed by cooling to ambient temperature, at a rate $5{ }^{\circ} \mathrm{C} \mathrm{min}^{-1}$. The color of the resulting ceramic pellets was bright yellow.

For crystal structure characterization, the pellets were ground to ensure that the structure was representative of the bulk ceramic. Samples were then manually crushed into fine powders. X-ray powder diffraction (XRD) data were collected on a PANalytical X'Pert Pro diffractometer (PANalytical, Cambridge, UK), fitted with an $\mathrm{X}^{\prime}$ Celerator detector, in $\theta / \theta$ geometry using Ni-filtered $\mathrm{Cu}-\mathrm{K} \alpha$ radiation $(\lambda=1.5418$ 
$\AA$ ), over the $2 \theta$ range 10 to $100^{\circ}$ in steps of $0.0167^{\circ}$, with an effective count time of 300 s per step.

For electrical characterization, silver electrodes were painted on parallel faces of the ceramic disks. Temperature-dependent dielectric data were collected by measuring the capacitance and loss at different frequencies on heating, using an LCR meter (Agilent E4980A) connected to a computer-controlled temperature chamber. The d.c. field dependence of dielectric permittivity was measured using an LCR meter (TH 2828S, Changzhou, China) connected to a high voltage source (Keithley 6517). To measure electric displacement - electric field $(D-E)$ and current - electric field $(I-E)$ hysteresis loops, gold electrodes were sputtered on the two main surfaces of polished specimens (with thickness of $c a .0 .3 \mathrm{~mm}$ ). The measurement was carried out using a ferroelectric measurement system (NPL, Teddington, UK). ${ }^{21}$ The hysteresis loops were measured separately at low and high electrical fields to investigate the sequence of electric field-induced transitions. The differential permittivity $(d D / d E)$ was calculated from the $D-E$ loops.

\section{Results and Discussion}

The fitted XRD patterns of the studied $\mathrm{ABN} x$ compositions are given as supplementary information in Fig. S1, with the crystal and refinement parameters summarized in Table S1. The fits confirm that all samples were single phase, within the limits of the technique, with an average $\mathrm{Pbcm}$ structure, as seen in the unsubstituted parent phase $\mathrm{AgNbO}_{3} \cdot{ }^{12,13-15}$ The compositional variations of lattice parameters and cell volume are shown in Fig. 1. The lattice parameters $a, b$ and the unit cell volume generally decrease with increasing $x$-value, while the $c$-axis parameter shows a general increase with increasing level of substitution, consistent with solid solution formation. The $b / a$ cell parameter ratio shows two approximately linear regions, with a step between $x=0.015$ and 0.020 . This is reflected in the volume plot, which switches from a linear to a curved region at $x=0.020$. The volume decrease is consistent with substitution of $\mathrm{Ag}^{+}$by $\mathrm{Bi}^{3+}$ cations with smaller 
ionic radius. However, at these levels of substitution, the results cannot easily distinguish between the three main possible solid solution mechanisms:

$$
\begin{array}{ll}
\mathrm{Bi}_{2} \mathrm{O}_{3} \rightarrow 2 \mathrm{Bi}_{\mathrm{Ag}}{ }^{\bullet \bullet}+4 \mathrm{~V}_{\mathrm{Ag}}{ }^{\prime}+3 \mathrm{O}_{\mathrm{O}}{ }^{\mathrm{X}} & \text { A site cation vacancy } \\
\mathrm{Bi}_{2} \mathrm{O}_{3}+4 \mathrm{Nb}_{\mathrm{Nb}}{ }^{\mathrm{X}} \rightarrow 2 \mathrm{Bi}_{\mathrm{Ag}} \bullet+4 \mathrm{Nb}^{\mathrm{IV}}{ }_{\mathrm{Nb}}{ }^{\prime}+3 \mathrm{O}_{\mathrm{O}}{ }^{\mathrm{X}} & \text { B site reduction } \\
\mathrm{Bi}_{2} \mathrm{O}_{3} \rightarrow 2 \mathrm{Bi}_{\mathrm{Ag}}{ }^{\bullet \bullet}+2 \mathrm{O}_{\mathrm{i}}{ }^{\prime \prime}+\mathrm{O}_{\mathrm{O}}{ }^{\mathrm{X}} & \text { O interstitial }
\end{array}
$$

Fig. 2 shows the temperature dependence of the real and imaginary components of dielectric permittivity at $1 \mathrm{MHz}$. A number of dielectric anomalies are observed in the plots for ABN005 and can be attributed to a series of phase transitions similar to those observed in $\mathrm{AgNbO}_{3}{ }^{11,12}$ The dielectric anomalies at $c a .50^{\circ} \mathrm{C}$ and $c a .260{ }^{\circ} \mathrm{C}$, are associated with transitions that maintain the $\left(a^{-}, b^{-}, c^{+}\right) /\left(a^{-}, b^{-}, c^{-}\right)$octahedral tilting system, viz.: $\mathrm{M}_{1} \leftrightarrow \mathrm{M}_{2}$ and $\mathrm{M}_{2} \leftrightarrow \mathrm{M}_{3}$ transitions, respectively. ${ }^{14,15}$ However, to date the structural details of these transitions are unclear. An extra anomaly was observed in the imaginary part of the dielectric permittivity $\left(\varepsilon_{\mathrm{r}}^{\prime \prime}\right)$ at $c a .170{ }^{\circ} \mathrm{C}$, which is assigned to the freezing temperature $\left(T_{f}\right)$, where the antipolar dipoles become frozen as the cations order. ${ }^{15,22}$ The sharp maximum near $350{ }^{\circ} \mathrm{C}$ and the shoulder near 400 ${ }^{\circ} \mathrm{C}$ are attributed to phase transitions between phases with different octahedral tilting systems, i.e. orthorhombic Pbcm $\left(\left(a^{-}, b^{-}, c^{+}\right) /\left(a^{-}, b^{-}, c^{-}\right)\right) \leftrightarrow$ orthorhombic Cmcm $\left(a^{0}, b^{-}, c^{+}\right) \leftrightarrow$ tetragonal $P 4 / m b m\left(a^{0}, b^{0}, c^{+}\right)$transitions, respectively. ${ }^{14}$ The temperatures corresponding to all these dielectric peaks are frequency independent (Fig. S2). As shown in Fig. 2, with increasing $x$, all the dielectric anomalies shift to lower temperature. The dielectric anomalies corresponding to the $\mathrm{M}_{1} \leftrightarrow \mathrm{M}_{2}$ transition and the $T_{f}$ are shifted below room temperature at compositions $x \geq 0.010$ and $x>0.020$, respectively.

Fig. 3 shows the variation of dielectric permittivity of $\mathrm{ABN} x$ ceramics under d.c. field. The dielectric permittivity of $\mathrm{ABN} x$ ceramics decreases with increasing d.c. field, as the system becomes less polarizable when it approaches saturation. For the $x$ 
$=0.005$ composition, there is a clear drop in dielectric permittivity at zero field, compared to the initial state, after initial application and removal of the d.c. field. This can be attributed to a decrease in FE domain wall density, in agreement with previous studies. $^{23}$ In contrast, at higher $x$-value compositions, there is no clear drop of permittivity after application and removal of the d.c. field, which suggests that these compositions have less polarizable structures. The d.c. field-induced variations of dielectric permittivity decrease with increasing $x$-value, consistent with the ceramics progressively evolving towards an antiferroelectric state. The ABN040 ceramic exhibits very stable dielectric permittivity under d.c. field compared to the other compositions, consistent with the structure being antiferroelectric. Similar results are seen in antiferroelectric $\mathrm{PbZrO}_{3}$, which also shows no variation in dielectric permittivity under a bias field, when this field is lower than the critical field required to induce the AFE-FE transformation. ${ }^{24}$

Fig. 4 shows the $D-E$ and $I-E$ loops of ABN $x$ ceramics up to $6 \mathrm{MV} \mathrm{m}^{-1}$. The loops relative to the ABN005 ceramic (Fig. 4a) can be interpreted based on the idea that the local polar regions and $\mathrm{FE}$ domains that might be initially present in $\mathrm{AgNbO}_{3},{ }^{12,25,26}$ are slightly diminished by low levels of bismuth substitution. The I-E loop of $\mathrm{AgNbO}_{3}$ in fact showed two peaks in the loading branch (indicated by $E_{1}=0.3 \mathrm{MV} \mathrm{m}^{-1}$ and $E_{2}$ $=2.5 \mathrm{MV} \mathrm{m}^{-1}$ in Ref.12), which can be attributed to complicated domain switching events relative to the local polar regions. Whilst these local polar regions appear to be ferroelectric, the possibility of them being ferrielectric in nature cannot be excluded. ${ }^{16}$ In ABN005, the current peaks are not clearly visible, in agreement with the view that bismuth addition hinders the presence of polar region (or reduces their polarity). However, a closer look at Fig. 4 a reveals the presence of a rather smeared and broad current bump (roughly $2 \mathrm{MV} \mathrm{m}^{-1}$, ie., $E_{2}$ ). This may correspond to a local electric field-induced transition, which would expand the polar regions (or increase their polarity). The current peaks $E_{1}$, near zero field could therefore contain the contribution of the reversed transition occurring during unloading, and the contribution of the domain switching event that in $\mathrm{AgNbO}_{3}$ has been associated with the peak $E_{1}$ (during loading), which here might be responsible for the tail of the 
current peak appearing in the loading branch. With increasing $x$-value, the $E_{2}$ peak in $\mathrm{ABN} x$ ceramics becomes weaker and broader, when $T_{\mathrm{f}}$ is below room temperature. The composition ABN040 shows linear dielectric behavior with no presence of domain switching peaks in the $I-E$ loop (Fig. 4b), which further supports the proposition that the structure of this composition is antiferroelectric. This is consistent with the observation that the $E_{2}$ peak completely disappears when $T_{\mathrm{f}}$ is below room temperature.

Fig. 5 shows the high field $D-E$ loops and $\mathrm{d} D / \mathrm{d} E-E$ loops of the studied $A B N x$ ceramics. As the $x$-value increases, the field-induced ferroelectric polarization decreases (Fig. 5a), with the maximum electric displacement, $D_{\mathrm{m}}$, decreasing from $0.40 \mathrm{C} \mathrm{m}^{-2}$ to $0.23 \mathrm{C} \mathrm{m}^{-2}$ at the highest applied field, while the remnant electric displacement $\left(D_{\mathrm{r}}\right)$ becomes almost zero at $x=0.030$. At $x=0.040$, a linear-like $D-E$ hysteresis is observed. In Fig. $5 \mathrm{~b}, \pm E_{\mathrm{F}}$ represents the electric forward field required to transform the initial structures to a ferroelectric state, while the backward field required to reverse the transition is denoted as $\pm E_{\mathrm{B}}$. All ceramics show double $D-E$ loops and four peaks in their $\mathrm{d} D / \mathrm{d} E-E$ loops. Additionally, two small peaks (denoted as $\left.\pm \mathrm{E}_{\mathrm{U}}\right)$ are observed in compositions with $x<0.020$. Analogous peaks were observed in ferroelectric loops for pure $\mathrm{AgNbO}_{3}$ ceramic, ${ }^{10}$ which were attributed to a polarizable ferrielectric-like structure below $T_{f}$. In contrast, there are no clear $\pm E_{\mathrm{U}}$ peaks in the $\mathrm{d} D / \mathrm{d} E-E$ loops of ABN $x$ ceramics with $x \geq 0.020$ (Fig. $4 \mathrm{~b}$ ); this supports the idea that the structure above $T_{f}$ is non-polarizable antiferroelectric (see Fig. 2). Additionally, the results suggest that with increasing $x$-value, the local polar ferroelectric or ferrielectric behavior is suppressed and that the AFE structure becomes more favored at room temperature, with a reduced ability to be polarized under the application of an electric field.

Fig. 6 shows the compositional variation of $E_{\mathrm{F}}$ and $E_{\mathrm{B}}$. Both $E_{\mathrm{F}}$ and $E_{\mathrm{B}}$ shift to higher field with increasing $x$-value. This increase can lead to higher energy density. Moreover, the difference $(\Delta E)$ between $E_{\mathrm{F}}$ and $E_{\mathrm{B}}$ decreases with increasing $x$-value, which can result in improved energy storage efficiency. The schematic energy storage process of an ideal antiferroelectric capacitor is shown in Fig. 7a, where recoverable 
energy density $\left(W_{\text {rec }}\right)$ and energy loss $\left(W_{\text {loss }}\right)$ are represented by the red and black areas, respectively. The energy efficiency $(\eta)$ can be calculated as:

$$
\eta=W_{\text {rec }} /\left[W_{\text {loss }}+W_{\text {rec }}\right]
$$

The calculated energy storage density and efficiency of the studied ABN $x$ ceramics is shown in Fig. 7b. The maximum recoverable energy density $W_{\text {rec. }}\left(3 \mathrm{~J} \mathrm{~cm}^{-3}\right)$ was achieved in the ABN010 composition, as a result of the favorable balance between $D_{m}$ (which decreases with increasing $x$-value) and the increased electric field required to induce the reversible phase transitions (which increases with increasing $x$-value). The optimal energy storage performance (with a high recoverable energy density $W_{\text {rec }}$ of $2.6 \mathrm{~J} \mathrm{~cm}^{-3}$ and high energy efficiency of 0.86) was obtained in the ABN030 ceramic. The ceramics $\mathrm{ABN} 010$ and $\mathrm{ABN} 030$ show higher recoverable energy density $W_{\text {rec }}$ than any other known lead free ceramics, as shown in Fig. 7c, which depicts the relationship between $W_{\text {rec }}$ and applied field $E$, for recently developed lead-free dielectric materials. The $\mathrm{AgNbO}_{3}$-based ceramics typically show high $W_{\text {rec }}$ values of $2 \sim 3 \mathrm{~J} \mathrm{~cm}^{-3}$ at low applied fields of around $200 \mathrm{kV} \mathrm{cm}^{-1}$. Among ABN ceramics, ABN030 offers the highest energy efficiency, $\eta$, as shown in Fig. 7b. The main benefit of bismuth substitution in the $\mathrm{ABN} x$ ceramics compared to the unsubstituted parent material is the significant increase in the efficiency, which is double that of pure $\mathrm{AgNbO}_{3}$ (Fig. 7b). The efficiency of $\mathrm{ABN030}$ is amongst the highest values found in lead-free ceramics (Fig. 7d).

\section{Conclusions}

Ceramics of composition $\mathrm{Ag}_{1-3 x} \mathrm{Bi}_{x} \mathrm{NbO}_{3}(0.005 \leq x \leq 0.040)$ were successfully prepared by solid state methods. The effect of bismuth substitution is to suppress the formation of local polar regions, to induce antiferroelectric order and to increase stability under electric field loading. Increasing levels of bismuth substitution also leads to a progressive decrease of the temperatures associated with the $M_{1}$ to $M_{2}$ and 
$\mathrm{M}_{2}$ to $\mathrm{M}_{3}$ phase transitions and of the freezing temperature $T_{f}$. Above $T_{f}$, the structure of the $\mathrm{M}_{2}$ phase is AFE, while below $T_{f}$ the structure contains local polar regions and is polarizable. The hysteresis loop measurements indicated that the domain switching events found in the $\mathrm{M}_{1}$ phase of $\mathrm{AgNbO}_{3}$ are suppressed for low substitution levels. The presence of electric field induced antiferroelectric to ferroelectric transitions is increasingly hindered with increasing bismuth content. The difference between the $\mathrm{M}_{1}$ and the $\mathrm{M}_{2}$ phases below $T_{f}$ is that while the $\mathrm{M}_{1}$ phase has locally stabilized ferroelectric domain structure, the $\mathrm{M}_{2}$ phase only exhibits local polar regions. The difference between forward and back field decreases with increasing $x$-value leading to higher energy efficiency compared to unsubstituted $\mathrm{AgNbO}_{3}$. An optimal energy storage performance with a high energy storage density of $2.6 \mathrm{~J} \mathrm{~cm}^{-3}$ and high energy efficiency of 0.86 was achieved in the composition $\mathrm{Ag}_{0.91} \mathrm{Bi}_{0.03} \mathrm{NbO}_{3}$.

\section{Acknowledgements}

This work was supported by the National Basic Research Program of China (973Program) under Grant No. 2015CB654602, the International Science \& Technology Cooperation Program of China (Grant No. 2013DFR50470), the Natural Science Basis Research Plan in Shaanxi Province of China (Grant No. 2015JM5199), the Fundamental Research Funds for the Central Universities, and "111" Project (No. B14040). Ye Tian gratefully acknowledges financial support from the China Scholarship Council.

\section{References}

1. Q. Li, L. Chen, M. R. Gadinski, S. Zhang, G. Zhang, H. Li, A. Haque, L. Q. Chen, T. Jackson and Q. Wang, Nature, 2015, 523, 576

2. Z. Dang, J. Yuan, S. Yao and R. Liao, Adv. Mater., 2013, 25,6334

3. L. Jin, F. Li and S. Zhang, J.Am.Ceram.Soc.2014, 97, 1.

4. Y. Zhao, X. Hao, Q. Zhang, ACS Appl. Mater. Interfaces, 2014, 6, 11633-11639.

5. B. Peng, Qi. Zhang, X. Li, T. Sun, H. Fan, S. Ke, M. Ye, Y. Wang, W. Lu, H. Niu, X. Zeng and 
H. Huang, ACS Appl. Mater. Interfaces, 2015, 7, 13512.

6. L. Jin, R. Huo, R. Guo, F. Li, D. Wang, Y. Tian, Q. Hu, X. Wei, Z. He, Y. Yan, and G. Liu, ACS Appl. Mater. Interfaces, 2016, 8, 31109-31119.

7. Y. Tian, X. Chao, L.Jin, L.Wei, P. Liang, and Z. Yang, Appl. Phys. Lett., 2014, 104, 112901.

8. X. Wei, H. Yan, T. Wang, Q. Hu, G. Viola, S. Grasso, Q. Jiang, L. Jin, Z. Xu and M. J. Reece, J. Appl. Phys., 2013, 113, 024103.

9. F. Li, J. Zhai, B. Shen, X. Liu, K. Yang, Y. Zhang, P. Li, B. Liu, and H. Zeng, J. Appl. Phys. 2017. 121, 054103.

10. L. Zhao, Q. Liu, S. Zhang and J. Li, J. Mater. Chem. C, 2016, 4, 8380.

11. D. Fu, M. Endo, H. Taniguchi, T. Taniyama and M. Itoh, Appl. Phys. Lett., 2007, 90, 252907.

12. Y. Tian, L. Jin, H. Zhang, Z. Xu, X.Wei, E. D. Politova, S. Yu. Stefanovich, Nadezda V. Tarakina, I.Abrahams and H.Yan , J. Mater. Chem. A, 2016,4, 17279-17287

13. J. Fábry, Z. Zikmund, A. Kania and V. Petrícek, Acta Crystallogr. C, 2000, 56, 916.

14. P. Sciau, A. Kania, B. Dkhil, E. Suard and A. Ratuszna, J. Phys.: Condens. Matter, 2004, 16, 2795.

15. I. Levin, V. Krayzman, J. C. Woicik, J. Karapetrova, T. Proffen, M. G. Tucker and I. M. Reaney, Phys. Rev. B: Condens. Matter Mater. Phys., 2009, 79, 104113.

16. M. Yashima, S. Matsuyama, R. Sano, M. Itoh, K. Tsuda and D. Fu, Chem. Mater, 2011, 23, 1643.

17. H. Moriwake, A. Konishi, T. Ogawa, C. A. J. Fisher, A. Kuwabara and D. Fu, J. Appl. Phys., 2016, 119, 064102.

18. W. G. Mumme, I. E. Grey, B. Edwards, C. Turner, J. Nino and T. A. Vanderah, J. Soli. Stat. Chem.2013, 200, 323-327.

19. R. D. Shannon, Acta Cryst. 1976, A32, 751.

20. H. Shimizu, H. Guo, S. E. Reyes-Lillo, Y. Mizuno, Karin M. Rabe and C. A. Randall, Dalton Trans., 2015, 44, 10763.

21. H. Yan, F. Inam, Giuseppe, Viola, H. Ning, H. Zhang, Q. Jiang, T. Zeng, Z. Gao and M. J Reece, J. Adv. Dielectr. 2011, 1, 107118

22. A. Kania and J. Kwapuliński, J. Phys.: Condens. Matter, 1999, 11, 8933.

23. Y. Masuda, Jpn. J. Appl. Phys. 1994, 33, 5549. 
24. G. Shirane, E. Sawaguchi and Y. Takagi, Phys Review, 1951, 84, 476.

25. A. Kania, K. Roleder, and M. Łukaszewski, Ferroelectrics, 1984, 52, 265.

26. A. Kania, K. Roleder, Ferroelectric Lett.,1984, $2,51$.

27. Q. Hu, L. Jin, T. Wang, C. Li, Z. Xing and X. Wei, J. Alloys Compd., 2015, 640, 416.

28. T. Wang, L. Jin, C. Li, Q. Hu and X. Wei, J. Am. Ceram. Soc.,2015, 98, 559.

29. Z. Shen, X. Wang, B. Luo and L. Li, J. Mater. Chem. A, 2015, 3,18146.

30. Z. Song, H. Liu, S. Zhang, Z. Wang, Y. Shi, H. Hao, M. Cao, Z. Yao and Z. Yu, J. Eur. Ceram. Soc., 2014, 34, 1209.

31. J. Ding, Y. Liu, Y. Lu, H. Qian, H. Gao, H. Chen and C. Ma, Mater. Lett., 2014, 114, 107.

32. L. Luo, B. Wang, X. Jiang and W. Li, J. Mater. Sci., 2014, 49,1659.

33. Q. Xu, T. Lia, H. Hao, S. Zhang, Z. Wang, M. Cao, Z. Yao and H. Liu, J. Eur. Ceram. Soc., 2014, 34, 1209.

34. J. Ye, Y. Liu, Y. Lu, J. Ding, C. Ma, H. Qian and Z. Yu, J. Mater. Sci.: Mater. Electron., 2014, $25,4632$.

35. H. Borkar, V. N. Singh, B. P. Singh, M. Tomar, V. Gupta and A. Kumar, RSC Adv., 2014, 4, 22840.

36. B. Wang, L. Luo, X. Jiang, W. Li and H. Chen, J. Alloys Compd., 2014, 585, 14-18.

37. B. Qu, H. Du and Z. Yang, J. Mater. Chem. C, 2016, 4, 1795.

38. A. C. Larson and R. B. Von Dreele, Los Alamos National Laboratory Report, No. LAUR-86$748,1987$. 


\section{Figure and Figure Captions}

\section{Abstract Graphic}
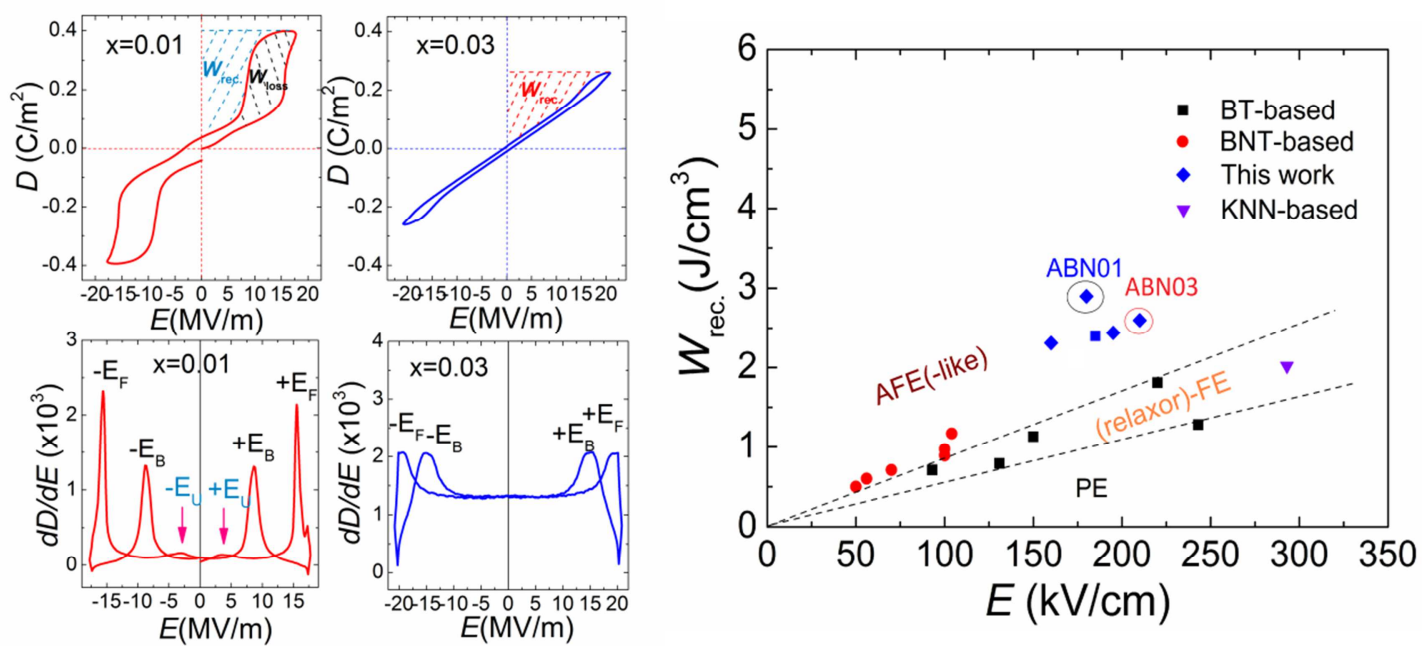

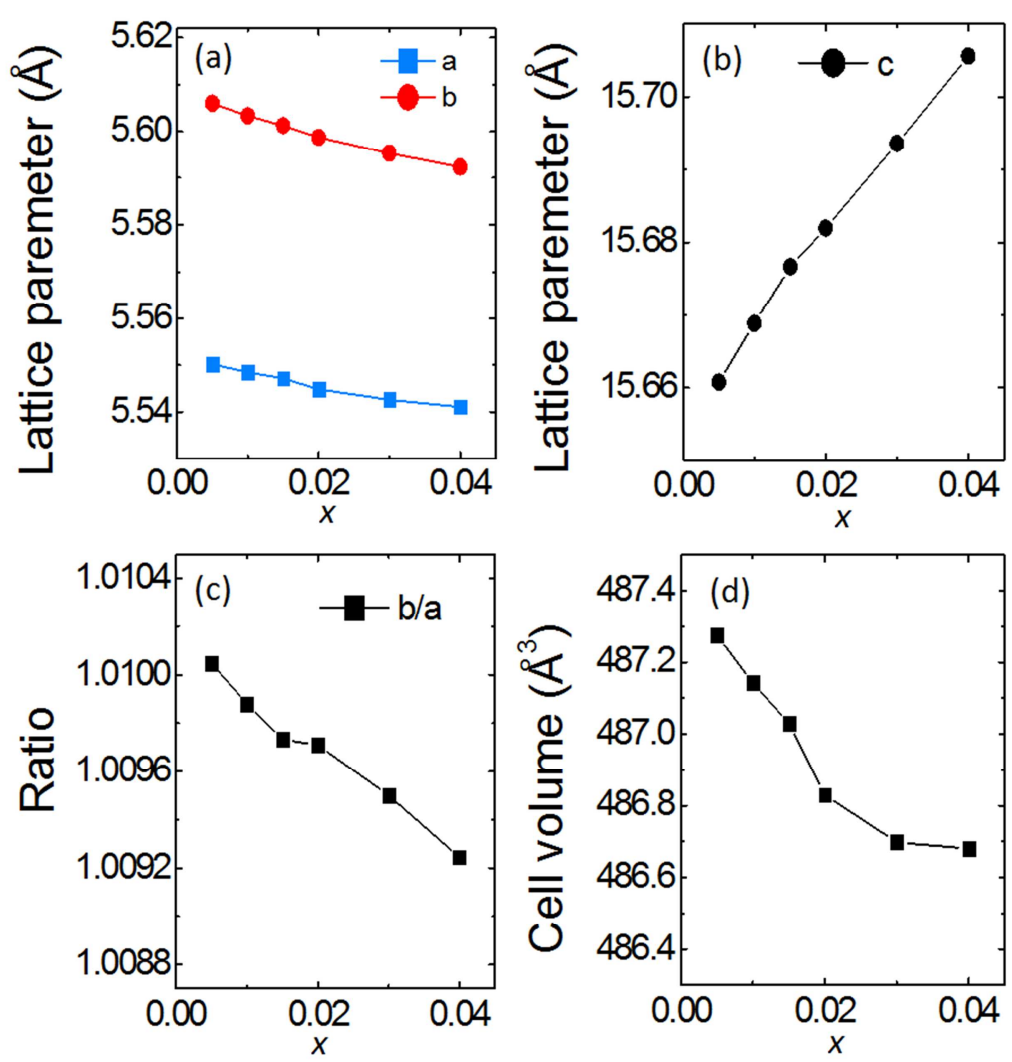

Fig.1. Compositional variation of (a) $a$ and $b$ lattice parameters (b) $c$ lattice parameter (c) b/a lattice parameter ratio and (d) unit cell volume in $\mathrm{ABN} x$ ceramics. 

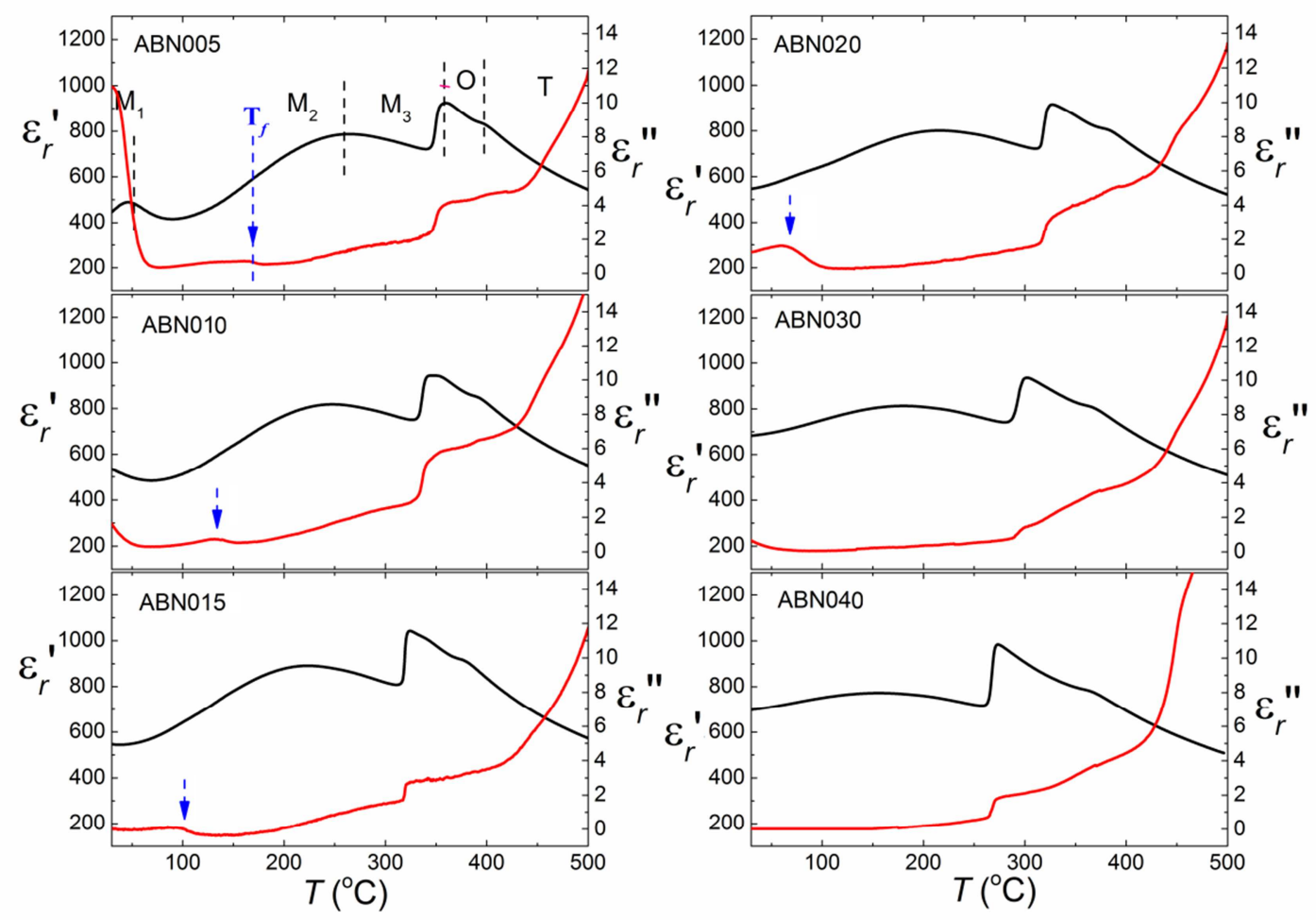

Fig. 2. Thermal dependence on heating of real $\left(\varepsilon_{r}^{\prime}\right)$ and imaginary $\left(\varepsilon_{r}{ }^{\prime \prime}\right)$ parts of relative permittivity in $\mathrm{ABN} x$ ceramics at $1 \mathrm{MHz}$. 

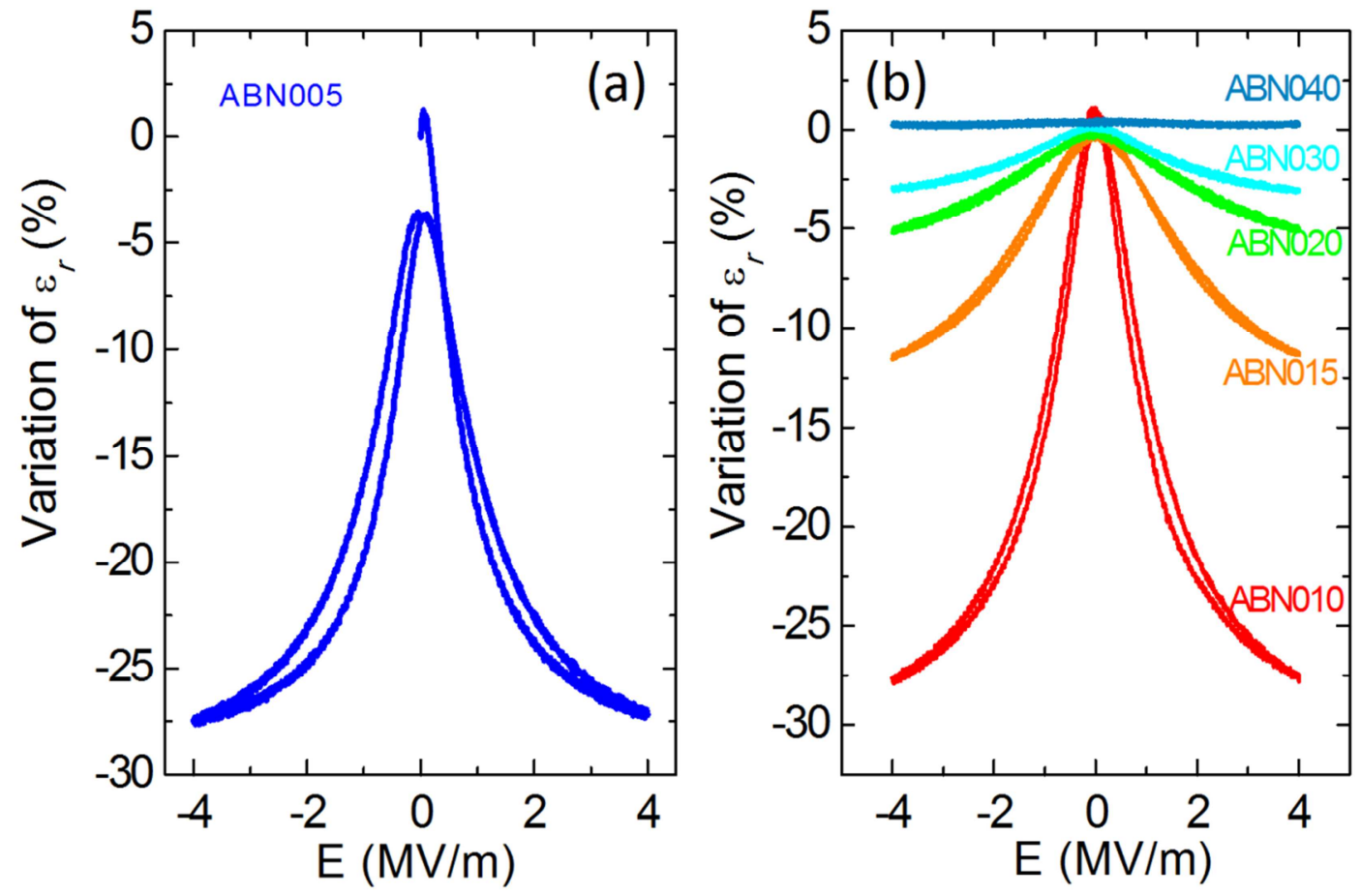

Fig. 3. Percentage variation in relative permittivity $\left(\varepsilon_{r}\right)$ at low field in $\mathrm{ABN} x$ ceramics measured at $1 \mathrm{kHz}$. 


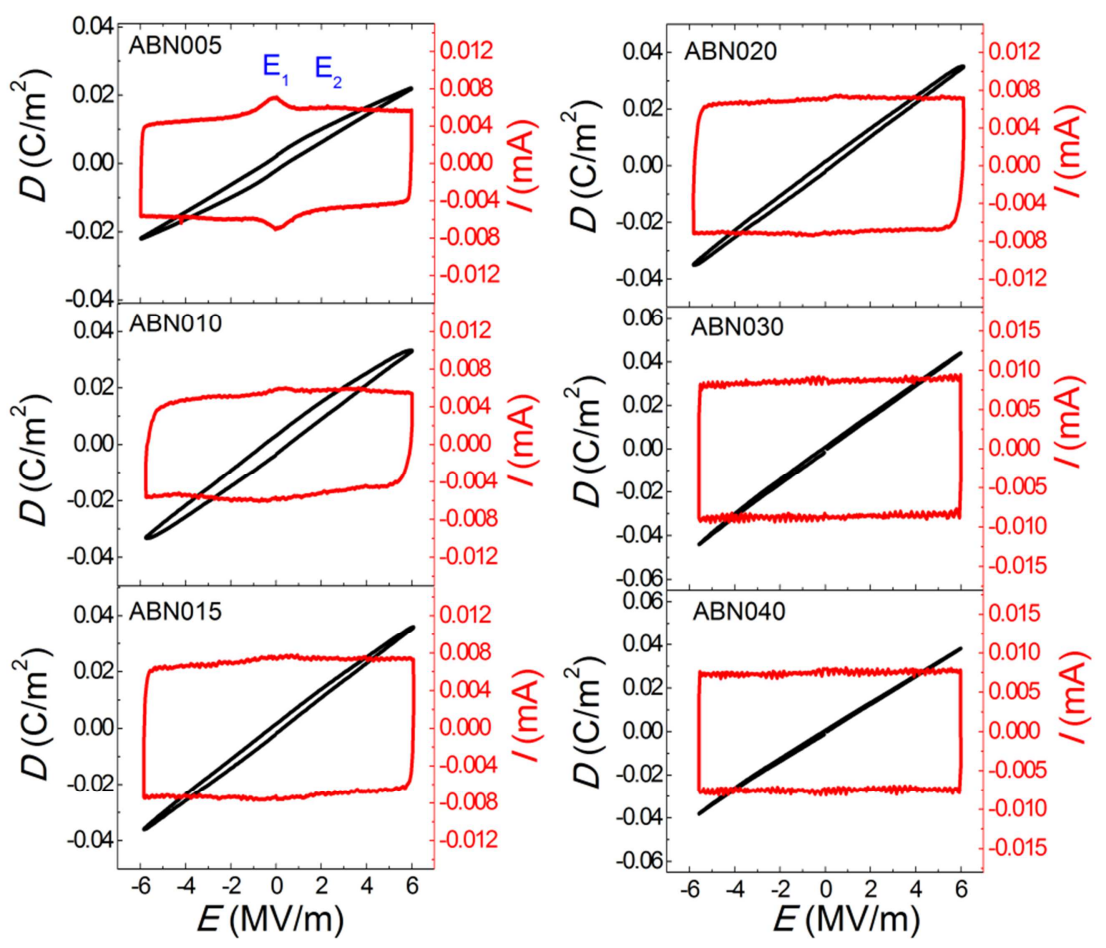

Fig. 4. Low field ferroelectric $D-E$ and $I-E$ loops measured at $10 \mathrm{~Hz}$ for $\mathrm{ABNx}$ ceramics. 
(a)
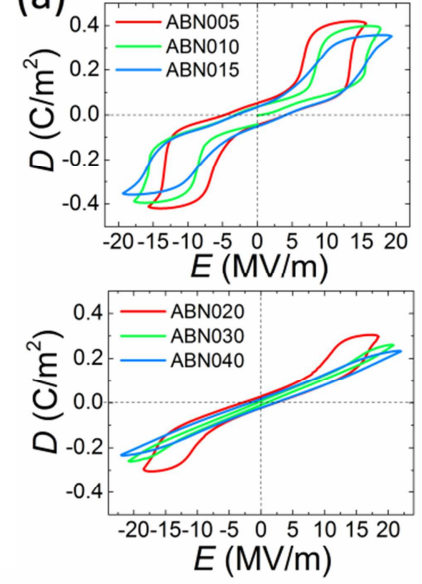

(b)
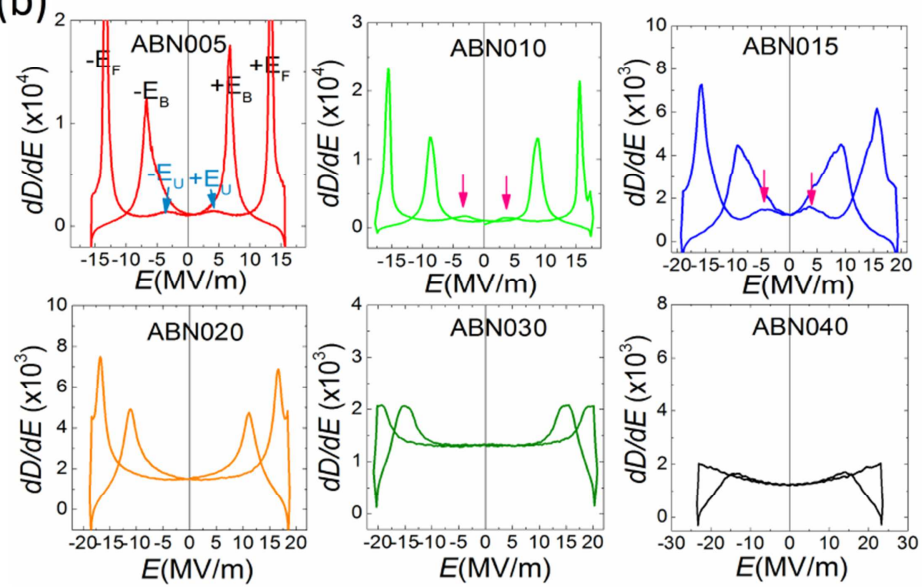

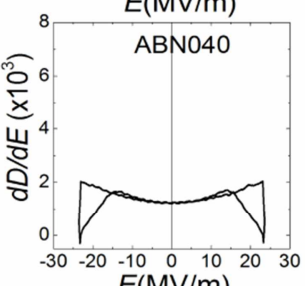

Fig. 5. High field ferroelectric (a) $D-E$ and (b) $d D / d E-E$ loops of $A B N x$ ceramics measured at $10 \mathrm{~Hz}$. 


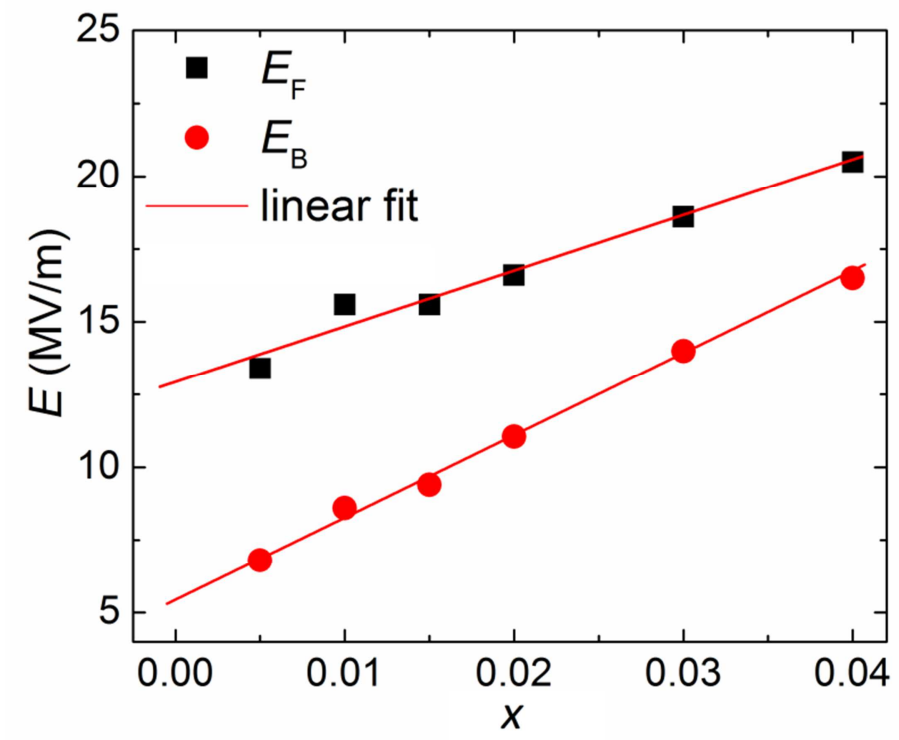

Fig. 6. Compositional variation of forward $\left(E_{\mathrm{F}}\right)$ and backward $\left(E_{\mathrm{B}}\right)$ field in $\mathrm{ABN} x$ ceramics. 

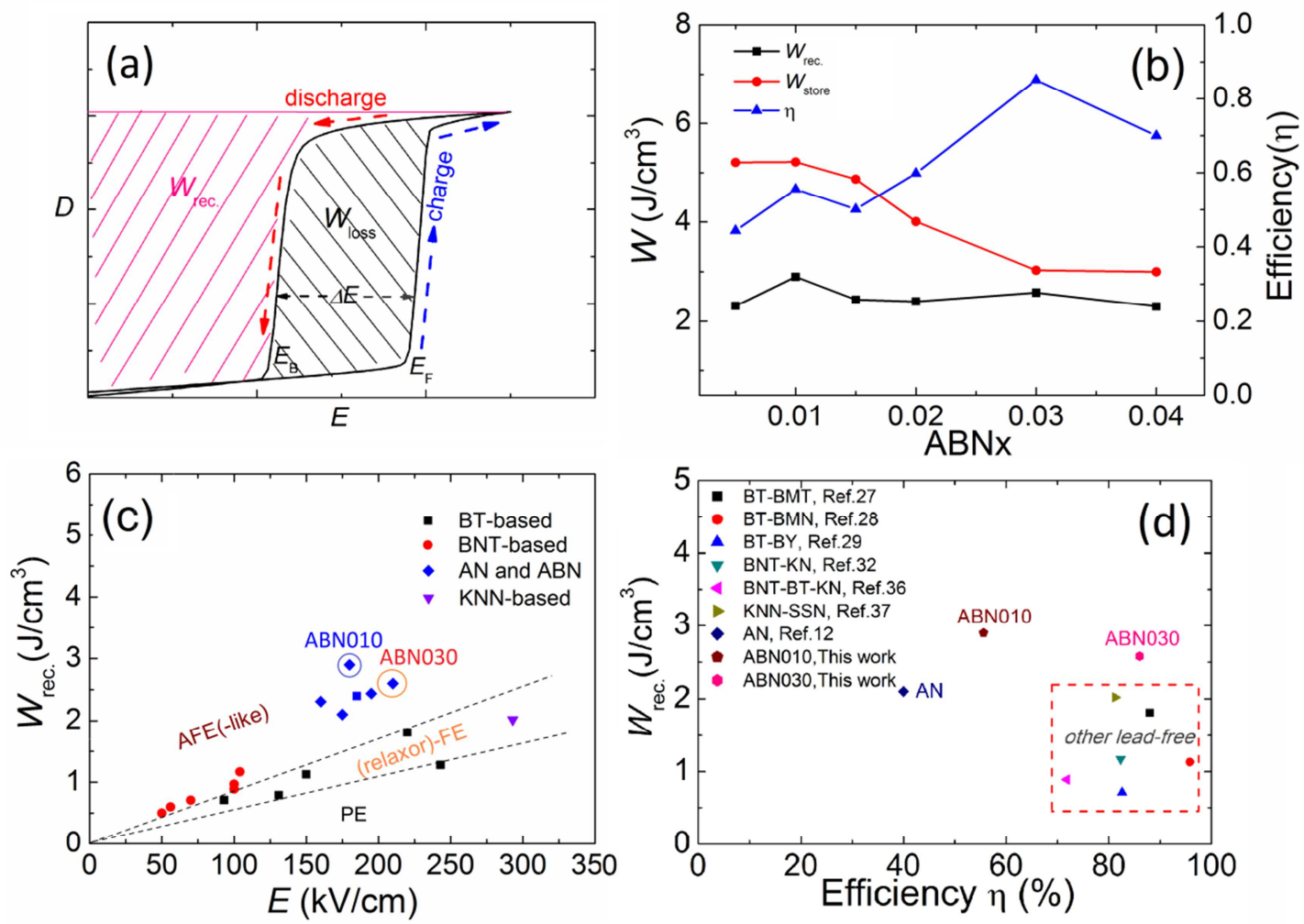

Fig. 7. (a) Schematic energy storage process for an ideal antiferroelectric capacitor; (b) energy storage density ( $W$ ) and efficiency $(\eta)$ of $\mathrm{ABN} x$ ceramics; (c) comparison of recoverable energy density $\left(W_{\text {rec }}\right)$ in $\mathrm{ABN010}$ and ABN030 with that in other lead-free ceramic systems: BT $\left(\mathrm{BaTiO}_{3}\right.$-based, $\mathrm{Hu}$ et al, ${ }^{27}$ Wang et al, ${ }^{28}$ Shen et al, ${ }^{29}$ and Song et $\left.a l,{ }^{30}\right)$; BNT $\left(\mathrm{Bi}_{0.5} \mathrm{Na}_{0.5} \mathrm{TiO}_{3}\right.$-based, Ding et al, ${ }^{31}$ Luo et al, ${ }^{32} \mathrm{Xu}$ et al, ${ }^{33} \mathrm{Ye}$ et $a l,{ }^{34}$ Borkar et al, ${ }^{35}$ and Wang et al, $\left.{ }^{36}\right)$; $\mathrm{KNN}\left(\mathrm{K}_{0.5} \mathrm{Na}_{0.5} \mathrm{NbO}_{3}\right.$-based. Qu et al, $\left.{ }^{37}\right)$ and (d) $W_{\text {rec }}$ versus $\eta$ for ABN030 ceramic compared to other lead-free ceramic systems (Tian et al, ${ }^{12} \mathrm{Hu}$ et $a l,{ }^{27}$ Wang et $a l,{ }^{28}$ Shen et $a l,{ }^{29}$ Luo et $a l,{ }^{32}$ Wang et al, ${ }^{36} \mathrm{Qu}$ et $\left.a l,{ }^{37}\right)$. 


\section{Supplementary Information}
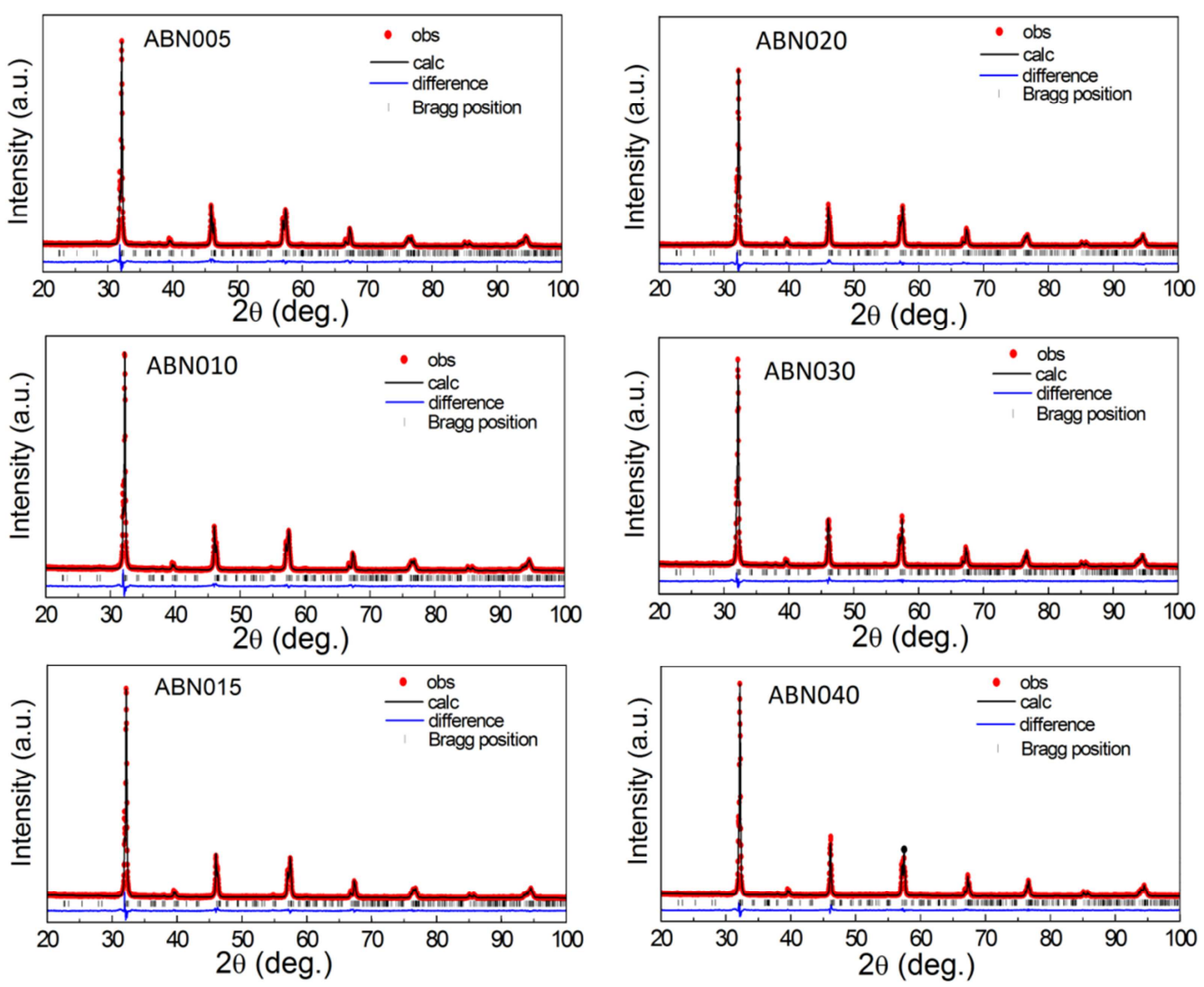

Fig. S1. Fitted X-ray diffraction profiles for crushed ABN $x$ ceramic powders. 

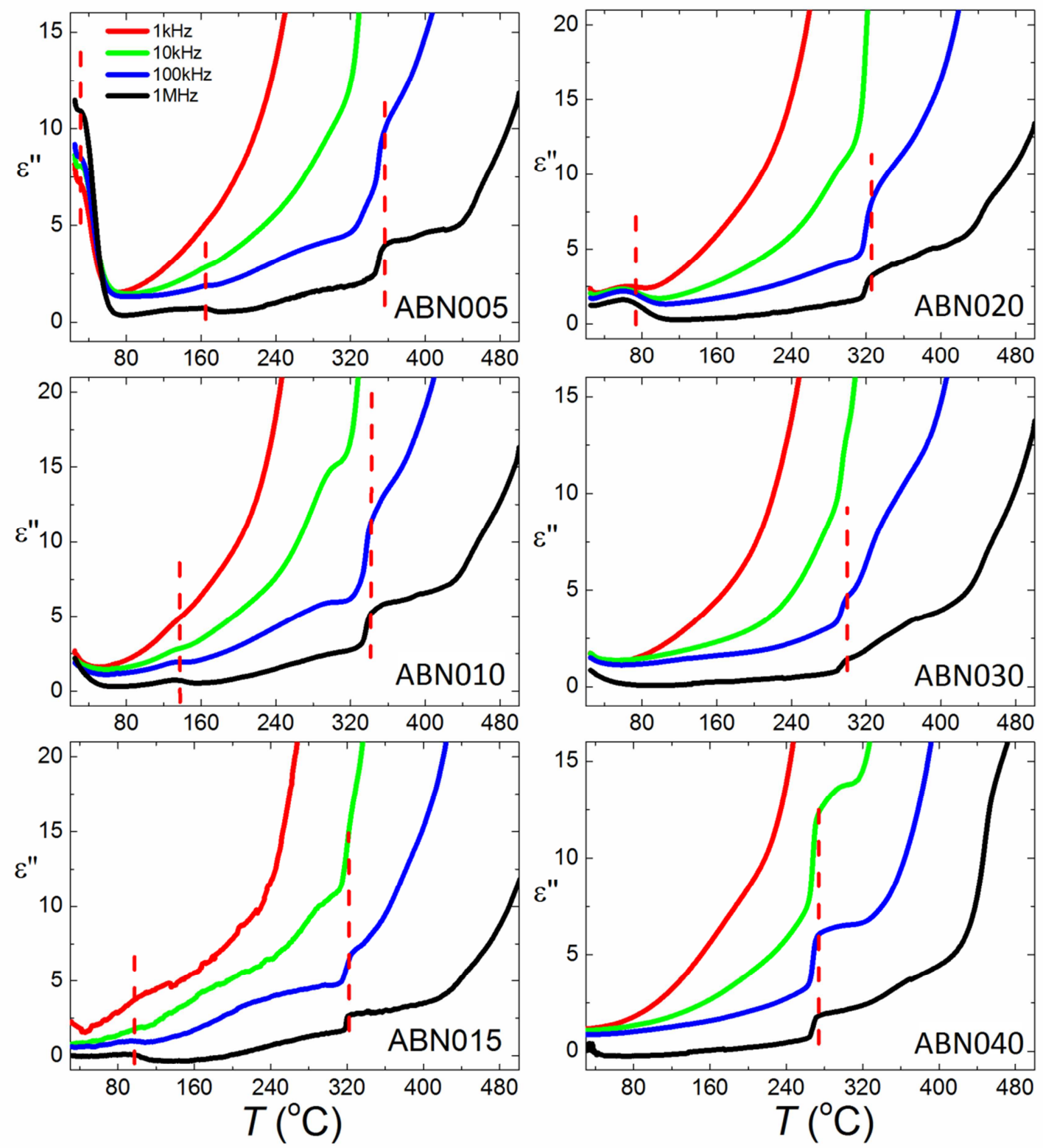

Fig. S2. Temperature dependence on heating of imaginary $\left(\varepsilon^{\prime \prime}\right)$ part of dielectric permittivity in $\mathrm{ABN} x$ ceramics. 
Table S1: Crystal and Refinement parameters for $\mathrm{Ag}_{1-3 x} \mathrm{Bi}_{x} \mathrm{NbO}_{3}$. Estimated standard deviations are given in parentheses.

\begin{tabular}{|c|c|c|c|c|c|c|}
\hline Chemical formula & $\mathrm{Ag}_{0.985} \mathrm{Bi}_{0.005} \mathrm{NbO}_{3}$ & $\mathrm{Ag}_{0.97} \mathrm{Bi}_{0.01} \mathrm{NbO}_{3}$ & $\mathrm{Ag}_{0.955} \mathrm{Bi}_{0.015} \mathrm{NbO}_{3}$ & $\mathrm{Ag}_{0.94} \mathrm{Bi}_{0.02} \mathrm{NbO}_{3}$ & $\mathrm{Ag}_{0.91} \mathrm{Bi}_{0.03} \mathrm{NbO}_{3}$ & $\mathrm{Ag}_{0.88} \mathrm{Bi}_{0.04} \mathrm{NbO}_{3}$ \\
\hline Formula weight & $248.20 \mathrm{~g} \mathrm{~mol}^{-1}$ & $257.62 \mathrm{~g} \mathrm{~mol}^{-1}$ & $247.05 \mathrm{~g} \mathrm{~mol}^{-1}$ & $246.48 \mathrm{~g} \mathrm{~mol}^{-1}$ & $245.33 \mathrm{~g} \mathrm{~mol}^{-1}$ & $244.19 \mathrm{~g} \mathrm{~mol}^{-1}$ \\
\hline Crystal system & Orthorhombic & Orthorhombic & Orthorhombic & Orthorhombic & Orthorhombic & Orthorhombic \\
\hline Space group & $\mathrm{Pbcm}$ & Pbcm & $\mathrm{Pbcm}$ & $\mathrm{Pbcm}$ & $P b c m$ & $\mathrm{Pbcm}$ \\
\hline \multirow[t]{3}{*}{ Unit cell dimensions } & $a=5.5502(2) \AA$ & $a=5.5484(2) \AA$ & $a=5.5471(2) \AA$ & $a=5.5448(2) \AA$ & $a=5.5426(1) \AA$ & $a=5.5411(1) \AA$ \\
\hline & $b=5.6059(2) \AA$ & $b=5.6033(2) \AA$ & $b=5.6011(2) \AA$ & $b=5.5986(2) \AA$ & $b=5.5952(1) \AA$ & $b=5.5923(1) \AA$ \\
\hline & $c=15.6607(5) \AA$ & $c=15.6688(5) \AA$ & $c=15.6765(5) \AA$ & $c=15.6819(5) \AA$ & $c=15.6935(4) \AA$ & $c=15.7055(3) \AA$ \\
\hline Volume & $487.27(3) \AA^{3}$ & $487.14(3) \AA^{3}$ & $487.08(3) \AA^{3}$ & $486.83(3) \AA^{3}$ & $486.69(2) \AA^{3}$ & $486.68(2) \AA^{3}$ \\
\hline $\mathrm{Z}$ & 8 & 8 & 8 & 8 & 8 & 8 \\
\hline Density(calculated) & $6.766 \mathrm{~g} \mathrm{~cm}^{-3}$ & $6.753 \mathrm{~g} \mathrm{~cm}^{-3}$ & $6.738 \mathrm{~g} \mathrm{~cm}^{-3}$ & $6.724 \mathrm{~g} \mathrm{~cm}^{-3}$ & $6.696 \mathrm{~g} \mathrm{~cm}^{-3}$ & $6.665 \mathrm{~g} \mathrm{~cm}^{-3}$ \\
\hline \multirow{4}{*}{ R-factors ${ }^{\mathrm{a}}$} & $R_{\mathrm{wp}}=0.0401$ & $R_{\mathrm{wp}}=0.0373$ & $R_{\mathrm{wp}}=0.0409$ & $R_{\mathrm{wp}}=0.0369$ & $R_{\mathrm{wp}}=0.0328$ & $R_{\mathrm{wp}}=0.0348$ \\
\hline & $R_{\mathrm{p}}=0.0282$ & $R_{\mathrm{p}}=0.0256$ & $R_{\mathrm{p}}=0.0289$ & $R_{\mathrm{p}}=0.0263$ & $R_{\mathrm{p}}=0.0243$ & $R_{\mathrm{p}}=0.0251$ \\
\hline & $R_{\mathrm{ex}}=0.0165$ & $R_{\mathrm{ex}}=0.0160$ & $R_{\mathrm{ex}}=0.0166$ & $R_{\mathrm{ex}}=0.0163$ & $R_{\mathrm{ex}}=0.0164$ & $R_{\mathrm{ex}}=0.0595$ \\
\hline & $R_{\mathrm{F}}^{2}=0.1274$ & $R_{\mathrm{F}}^{2}=0.1084$ & $R_{\mathrm{F}}^{2}=0.1757$ & $R_{\mathrm{F}}^{2}=0.1208$ & $R_{\mathrm{F}}^{2}=0.1081$ & $R_{\mathrm{F}}^{2}=0.1164$ \\
\hline Total No. of variables & 27 & 27 & 27 & 27 & 27 & 27 \\
\hline No. of profile points used & 3460 & 3460 & 3460 & 3460 & 3460 & 3460 \\
\hline
\end{tabular}

\footnotetext{
${ }^{\mathrm{a}}$ For definition of R-factors see ref.38.
} 